\title{
HIGH POWER WINDOW TESTS AT SLAC*
}

\author{
A.E. Vlieks, W.R.Fowkes, R.Loewen, E. Jongewaard, A. Menegat, S. Tantawi, \\ SLAC, USA \\ Y H. Chin, H. Mizuno, K. Takata S. Tokumoto, KEK, Tsukuba Japan \\ S. Kazakov-BINP, Protvino Russia \\ R. Lawrence. Ives, Jeff Neilson, Calabazas Creek Research Inc, USA
}

\begin{abstract}
The successful design of high power windows play an essential role in RF power source development in accelerator programs such as the Next Linear Collider (NLC). Different window design approaches have been developed at several institutions to develop a successful high power window design. Some of the design considerations include the utilization of low loss RF modes, multiple RF modes and different window materials.

In the past year some of these designs have been tested at SLAC using a pair of 50+ MW X-band klystrons as power sources. This permitted testing to power levels exceeding $100 \mathrm{MW}$ at pulse widths ranging from 0.15 to 1.5 microseconds. We will present results of these tests including their RF performance and their optical and $\mathrm{x}$-ray emission characteristics as a function of pulse width and power levels.
\end{abstract}

\section{INTRODUCTION}

Programs such as the Next Linear Collider program (NLC) will require high power microwave devices such as Klystrons for power sources. These devices must supply $75 \mathrm{MW}$ of pulsed RF power with pulse widths of 3 microseconds. Ceramic windows are used to isolate the Klystron vacuum from the external environment while transmitting this RF power, virtually without loss. This paper describes a series of high power tests to evaluate the performance of window designs from several institutions.

The principle sources of window failure fall within three main categories: Dielectric failure; thermal failure; and boundary failure.[1] In Dielectric failure, the bulk material cannot stand off the electric field gradients across the material and a puncture results. In thermal failure, excessive thermal gradients along the window surface cause the ceramic to crack. In boundary failure, imperfections along the ceramic metal interface cause high, localized field gradients. This causes localized hot spots, which also cause cracking due to thermal stresses.

We have tested four different window designs, each of which addressed one or more of these sources of window failure. All of these windows are of the traveling Wave (TW) design or nearly so. [2]. This design approach lowers the peak electric field at the ceramic surface and reduces RF losses within the ceramic compared with the half-wave resonant window design.

\section{EXPERIMENT}

The window tests were performed in the Klystron Test $\mathrm{Lab}$ at SLAC. The power from two X-band Klystrons was combined to supply up to $110 \mathrm{MW}$ power to the windows under test. A Photomultiplier tube (PMT) and a video camera were used to measure the light emitted from the windows as well as identify its location on the surfaces. This was made possible by installing viewports at the entrance and exit of the windows. In addition, X-ray measurements were made using plastic scintillators.

The general method of testing was similar for all window designs. Initially the RF pulse width was narrowed to $150 \mathrm{~ns}$. The power was then gradually increased to $100 \mathrm{MW}$. After reaching a stable $100 \mathrm{MW}$ operation, the power was reduced and the pulse width was widened to $300 \mathrm{~ns}$. The power was then raised to $100 \mathrm{MW}$ over a period of hours. This procedure was repeated with an ever-increasing pulse width until a maximum stable power and pulse width was reached.

\subsection{KEK Window designs}

Two different KEK window designs were tested.

The design strategy for these designs was to incorporate a multiplicity of modes, which, when taken together, nearly canceled each other at the periphery. In this way the metal/ceramic boundary would not play an important role in breakdown. The first "mixed-mode" window incorporated a combination of $\mathrm{TE}_{11}+\mathrm{TM}_{11}$ modes. It had a diameter of $5.3 \mathrm{~cm}$. The second window had a larger diameter, $6.4 \mathrm{~cm}$. This larger diameter permitted the window to operate at a lower peak electric field, for a given power level at the expense of increasing the number of allowed modes. The mode combination for this window was $\mathrm{TE}_{11}+\mathrm{TM}_{11}+\mathrm{TE}_{12}$. See [3] for further details. The time history for these windows is shown in figure 1 . The pulse width and power is automatically recorded every

\footnotetext{
* Work supported by Department of Energy contract DE-AC03-76SF00515
} 
two minutes of operation and the resultant plots represent a compression of this data.
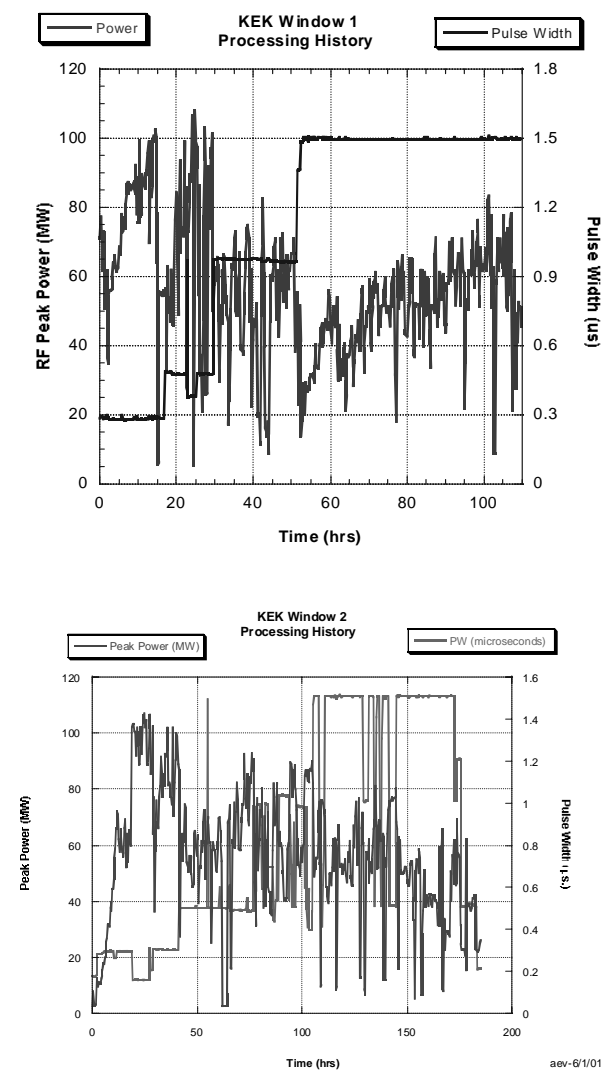

Figure 1. KEK Window Processing History

As can be seen from this figure, both windows successfully operated to the maximum power level (100 MW) for narrow pulse widths. At pulse widths of $1.5 \mu$ s however, both windows were limited to power levels of $\approx 80 \mathrm{MW}$. Figure 2 shows the effect of pulse width on light output as viewed by a PMT. Note that the light begins $\approx 150 \mathrm{~ns}$ after the RF. At the narrower pulse widths, where the window behaves quite stably, the light intensity is constant. However, as the pulse width is widened, the light intensity peaks. This is a measure of the level of conditioning of the window (its power handling capability). If the power were increas ed at the wider pulse widths the peak would rise steeply and breakdown would occur. However, running the window at a constant power level for a period of time resulted in the peak being reduced in amplitude. The power could then be increased with no breakdown. This technique became part of the conditioning procedure for this window. Further light curves may be found in ref. [3].

\subsection{SLAC Window design}

The SLAC window design [4] is the window currently used as the output window for the SLAC XL-4 Klystrons. It is an overmoded TW window operating in the $\mathrm{TE}_{01}$ mode. The competing $\mathrm{TE}_{02}$ mode is tuned out by a double step transition, whose dimensions are critical.

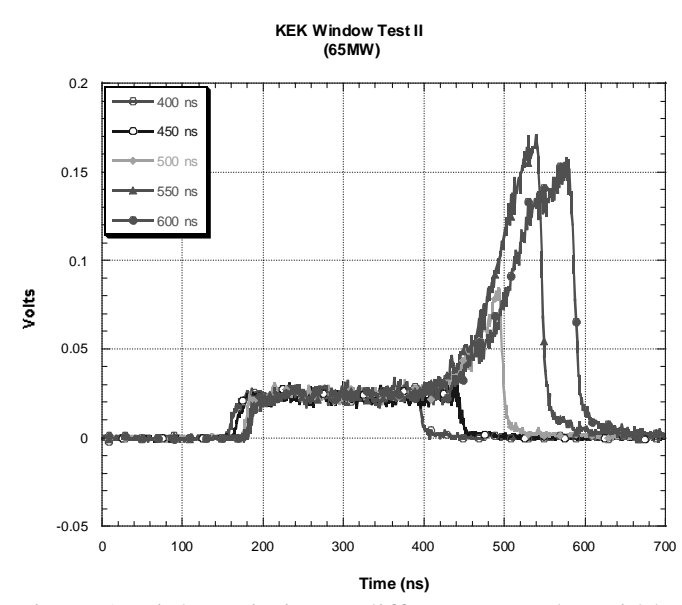

Figure 2. Light emission at different RF pulse widths

The SLAC Window was tested for $\approx 260$ hrs. During the first 200 hours of operation, testing was primarily at pulse widths $\leq 500 \mathrm{~ns}$ and power levels up to 100 MW. RF breakdown accompanied by vacuum bursts occurred frequently, as in the earlier KEK tests, but the unusual thing was that it occurred only on the downstream side of the window. (The window is symmetric). In order to determine whether there was a design flaw on one side of the window, the complete window assembly and mode converters were reversed with respect to power flow. The breakdown continued to be on the downstream side of the window indicating that it was linked to the flow of power. There is some indication that the breakdown is caused by trapped modes in the overmoded region of the window, (due to the mode converters), but this has not been conclusively verified. The total processing history is shown in figure 3.

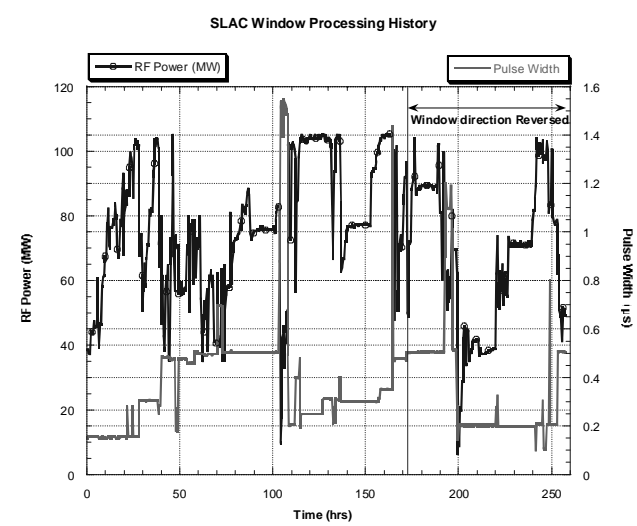

Figure 3. SLAC window Processing history

In figure 4, the light intensity is shown as a function of pulse width at $60 \mathrm{MW}$. As can be seen, the light intensity began $\approx 150 \mathrm{~ns}$ after the $\mathrm{RF}$ and then followed a constant but irregular intensity pattern. At $700 \mathrm{~ns}, \mathrm{RF}$ breakdown occurred. It is interesting to note that the light remained on for several microseconds during breakdown. The reason for this is unclear, but is probably linked to the creation of a plasma at 
breakdown or a local hot spot which requires time to cool.

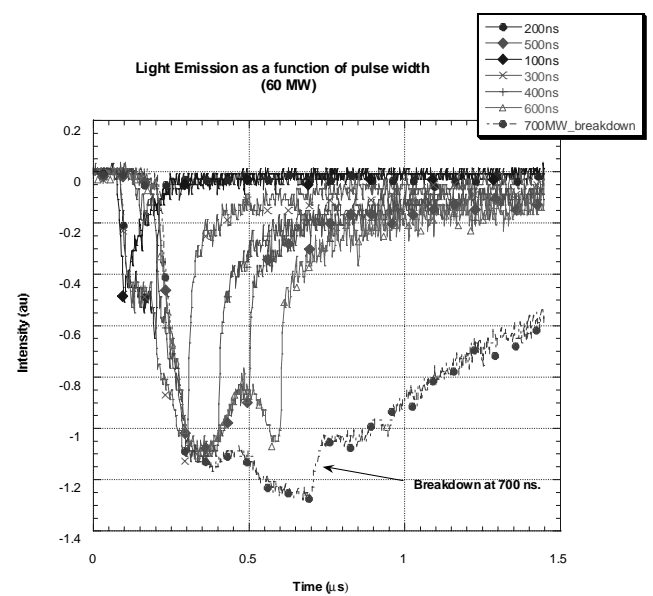

Figure 4. Light Emission as a function of pulse width

\subsection{Calabazas Creek (CC) Window design}

The principle goal in this design was to compare the performance of a high thermal conductivity, low loss Sapphire window with the more conventional Alumina windows. The RF design was modeled after the original SLAC $\mathrm{TE}_{01} \mathrm{TW}$ window. Figure 5 shows the processing history of this window.

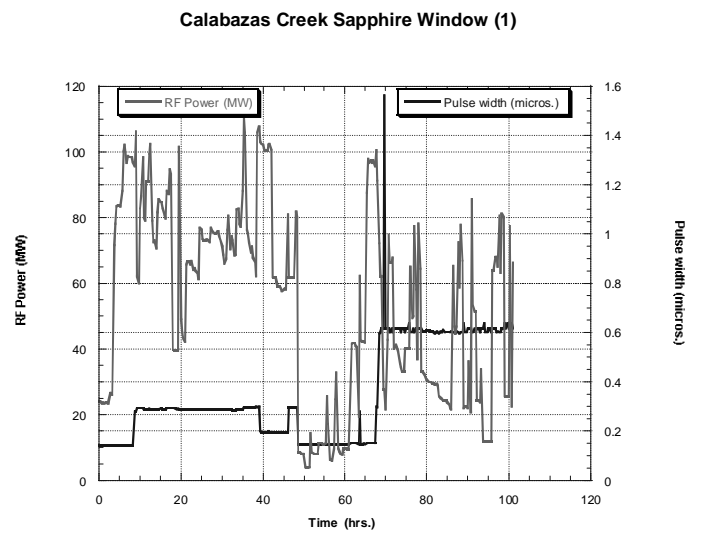

Figure 5.CC Window Processing History

This window initially processed very quickly, with no major breakdown events for the first 40 hours. Unfortunately it then suffered a major breakdown event, which caused a large "blemish" to appear on the window. Figure 6. shows the "doughnut" shaped visible light pattern at the TE01 intensity maximum. The blemish is also visible. This location became a source of breakdown (As observed by a video camera) making it impossible to run at $100 \mathrm{MW}$ for pulse widths greater than $150 \mathrm{~ns}$. A second blemish appeared later in the processing. On autopsy, the blemishes turned out to be localized surface melting.

\section{SUMMARY/CONCLUSIONS}

These measurements indicate that all four window designs can adequately handle RF power of up to 100
MW at narrow pulse widths. The KEK and SLAC designs can run at $100 \mathrm{MW}$ for $500 \mathrm{~ns}$ pulses. Even though these windows suffered numerous breakdown events, no lasting deterioration in performance was noted. The CC window handled $300 \mathrm{~ns}$ at $100 \mathrm{MW}$ but was damaged by its first major breakdown event. Later it ran at $80 \mathrm{MW}$ with $600 \mathrm{~ns}$ pulses

The KEK designs have run up to $\approx 80 \mathrm{MW}$ at pulse widths of $1.5 \mu \mathrm{s}$.

The SLAC window has run briefly at $65 \mathrm{MW}$ with $1.5 \mu$ s pulse widths.

Further measurements are required to determine the maximum power handling capability of these windows for $3 \mu$ s pulses.

All window designs radiate blue light during operation. The light intensity and pulse shape provides information on overall window performance and robustness.

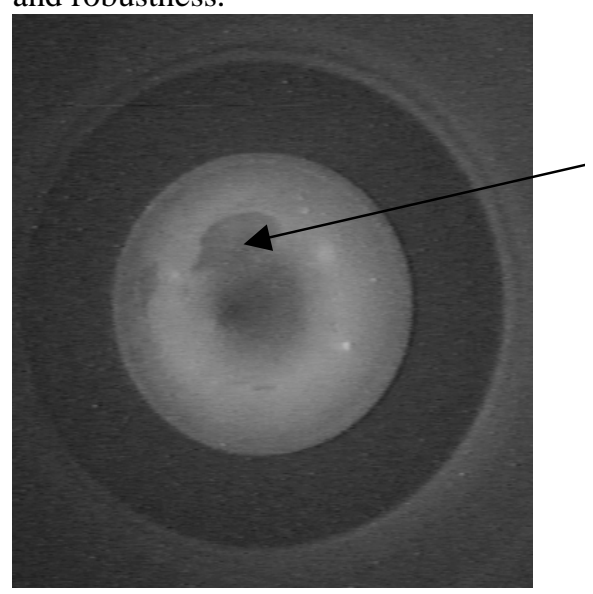

Figure 6. CC Sapphire window in operation.

\section{REFERENCES}

[1] A.E.Vlieks et Al, "Breakdown Phenomena in High Power Klystrons," XIIIth Int. Symposium on Discharges and Electric Insulation in Vacuum, Paris France, June27-30, 1988, and SLAC-Pub-4546 March 1988

[2] W. R. Fowkes et Al, "Reduced Field TE01 X-Band Traveling Wave Window", PAC 95 Dallas TX, 5/1$5 / 95$

[3] S. Tokumoto et Al, "High Power Testing Results of the X-Band Mixed-Mode RF Windows For Linear Colliders", LINAC 2000, Monterey CA, USA, Aug 21-25, 2000

[4] W.R. Fowkes et Al, "Recent Advances in High Power Windows at X-Band", High Density Microwaves, AIP Conf. Proc. 474, Pajaro Dunes, CA Oct. 1998. 